\title{
Sub-Riemannian structures on 3D Lie groups
}

\author{
Andrei Agrachev \\ SISSA, Trieste, Italy and MIAN, Moscow, Russia - agrachev@sissa.it \\ Davide Barilari \\ SISSA, Trieste, Italy - barilari@sissa.it
}

June 5,2018

\begin{abstract}
We give the complete classification of left-invariant sub-Riemannian structures on three dimensional Lie groups in terms of the basic differential invariants introduced in [4, 5]. This classifications recovers other known classification results in the literature, in particular the one obtained in 13 in terms of curvature invariants of a canonical connection. Moreover, we explicitly find a sub-Riemannian isometry between the nonisomorphic Lie groups $S L(2)$ and $A^{+}(\mathbb{R}) \times S^{1}$, where $A^{+}(\mathbb{R})$ denotes the group of orientation preserving affine maps on the real line.
\end{abstract}

MSC classes: $53 \mathrm{C} 17 \cdot 22 \mathrm{E} 30 \cdot 49 \mathrm{~J} 15$

Keywords: sub-Riemannian geometry, Lie groups, left-invariant structures.

\section{Introduction}

In this paper, by a sub-Riemannian manifold we mean a triple $(M, \Delta, \mathbf{g})$, where $M$ is a connected smooth manifold of dimension $n, \Delta$ is a smooth vector distribution of constant rank $k<n$, and $\mathbf{g}$ is a Riemannian metric on $\Delta$, smoothly depending on the point.

In the following we always assume that the distribution $\Delta$ satisfies the bracket generating condition (also known as Hörmander condition), i.e. the Lie algebra generated by vector fields tangent to the distribution spans at every point the tangent space to the manifold.

Under this assumption, $M$ is endowed with a natural structure of metric space, where the distance is the so called Carnot-Caratheodory distance

$$
\begin{array}{r}
d(p, q)=\inf \left\{\int_{0}^{T} \sqrt{\mathbf{g}_{\gamma(t)}(\dot{\gamma}(t), \dot{\gamma}(t))} d t \mid \gamma:[0, T] \rightarrow M\right. \text { is a Lipschitz curve, } \\
\left.\gamma(0)=p, \gamma(T)=q, \quad \dot{\gamma}(t) \in \Delta_{\gamma(t)} \text { a.e. in }[0, T]\right\} .
\end{array}
$$

As a consequence of the Hörmander condition this distance is always finite and continuous, and induces on $M$ the original topology (see Chow-Rashevsky Theorem, [7]). Standard references on sub-Riemannian geometry are [8, 15, 18].

A sub-Riemannian structure is said to be contact if its distribution is defined as the kernel of a contact differential one form $\omega$, i.e. $n=2 m+1$ and $\left(\bigwedge^{m} d \omega\right) \wedge \omega$ is a nonvanishing $n$-form on $M$. 
In this paper we focus on the three dimensional case. Three dimensional contact subRiemannian structures have been deeply studied in the last years (for example see [1, 5, 6]) and they have two basic differential invariants $\chi$ and $\kappa$ (see Section 3 for the precise definition and [2, 5] for their role in the asymptotic expansion of the sub-Riemannian exponential map).

The invariants $\chi$ and $\kappa$ are smooth real functions on $M$. It is easy to understand, at least heuristically, why it is natural to expect exactly two functional invariants. Indeed, in local coordinates the sub-Riemannian structure is defined by its orthonormal frame, i.e. by a couple of smooth vector fields on $\mathbb{R}^{3}$ or, in other words, by 6 scalar functions on $\mathbb{R}^{3}$. One function can be normalized by the rotation of the frame within its linear hull and three more functions by smooth change of variables. What remains are two scalar functions.

In this paper we exploit these local invariants to provide a complete classification of leftinvariant structures on 3D Lie groups. A sub-Riemannian structure on a Lie group is said to be left-invariant if its distribution and the inner product are preserved by left translations on the group. A left-invariant distribution is uniquely determined by a two dimensional subspace of the Lie algebra of the group. The distribution is bracket generating (and contact) if and only if the subspace is not a Lie subalgebra.

Left-invariant structures on Lie groups are the basic models of sub-Riemannian manifolds and the study of such structures is the starting point to understand the general properties of sub-Riemannian geometry. In particular, thanks to the group structure, in some of these cases it is also possible to compute explicitly the sub-Riemannian distance and geodesics (see in particular [14] for the Heisenberg group, 9] for semisimple Lie groups with Killing form and [17, 21] for a detailed study of the sub-Riemannian structure on the group of motions of a plane).

Remark. The problem of equivalence for several geometric structures close to left-invariant sub-Riemannian structures on 3D Lie groups were studied in several publications (see [10, 11, 13, 22, 23]). In particular in [22] the author provide a first classification of symmetric subRiemannian structures of dimension 3, while in [13] is presented a complete classification of sub-Riemannian homogeneous spaces (i.e., sub-Riemannian structures which admits a transitive Lie group of isometries acting smoothly on the manifold) by means of an adapted connection. The principal invariants used there, denoted by $\tau_{0}$ and $K$, coincide, up to a normalization factor, with our differential invariants $\chi$ and $\kappa$.

A standard result on the classification of 3D Lie algebras (see, for instance, [16]) reduce the analysis on the Lie algebras of the following Lie groups:

$H_{3}$, the Heisenberg group,

$A^{+}(\mathbb{R}) \oplus \mathbb{R}$, where $A^{+}(\mathbb{R})$ is the group of orientation preserving affine maps on $\mathbb{R}$,

$S O L V^{+}, S O L V^{-}$are Lie groups whose Lie algebra is solvable and has 2-dim square,

$S E(2)$ and $S H(2)$ are the groups of orientation preserving motions of Euclidean and Hyperbolic plane respectively,

$S L(2)$ and $S U(2)$ are the three dimensional simple Lie groups. 
Moreover it is easy to show that in each of these cases but one all left-invariant bracket generating distributions are equivalent by automorphisms of the Lie algebra. The only case where there exists two non-equivalent distributions is the Lie algebra $\mathfrak{s l}(2)$. More precisely a 2-dimensional subspace of $\mathfrak{s l}(2)$ is called elliptic (hyperbolic) if the restriction of the Killing form on this subspace is sign-definite (sign-indefinite). Accordingly, we use notation $S L_{e}(2)$ and $S L_{h}(2)$ to specify on which subspace the sub-Riemannian structure on $S L(2)$ is defined.

For a left-invariant structure on a Lie group the invariants $\chi$ and $\kappa$ are constant functions and allow us to distinguish non isometric structures. To complete the classification we can restrict ourselves to normalized sub-Riemannian structures, i.e. structures that satisfy

$$
\chi=\kappa=0, \quad \text { or } \quad \chi^{2}+\kappa^{2}=1 .
$$

Indeed $\chi$ and $\kappa$ are homogeneous with respect to dilations of the orthonormal frame, that means rescaling of distances on the manifold. Thus we can always rescale our structure in such a way that (1) is satisfied.

To find missing discrete invariants, i.e. to distinguish between normalized structures with same $\chi$ and $\kappa$, we then show that it is always possible to select a canonical orthonormal frame for the sub-Riemannian structure such that all structure constants of the Lie algebra of this frame are invariant with respect to local isometries. Then the commutator relations of the Lie algebra generated by the canonical frame determine in a unique way the sub-Riemannian structure.

Collecting together these results we prove the following

Theorem 1. All left-invariant sub-Riemannian structures on $3 D$ Lie groups are classified up to local isometries and dilations as in Figure 1, where a structure is identified by the point $(\kappa, \chi)$ and two distinct points represent non locally isometric structures.

Moreover

(i) If $\chi=\kappa=0$ then the structure is locally isometric to the Heisenberg group,

(ii) If $\chi^{2}+\kappa^{2}=1$ then there exist no more than three non isometric normalized subRiemannian structures with these invariants; in particular there exists a unique normalized structure on a unimodular Lie group (for every choice of $\chi, \kappa$ ).

(iii) If $\chi \neq 0$ or $\chi=0, \kappa \geq 0$, then two structures are locally isometric if and only if their Lie algebras are isomorphic.

In other words every left-invariant sub-Riemannian structure is locally isometric to a normalized one that appear in Figure 1, where we draw points on different circles since we consider equivalence classes of structures up to dilations. In this way it is easier to understand how many normalized structures there exist for some fixed value of the local invariants. Notice that unimodular Lie groups are those that appear in the middle circle (except for $A^{+}(\mathbb{R}) \oplus \mathbb{R}$ ).

From the proof of Theorem 1 we get also a uniformization-like theorem for "constant curvature" manifolds in the sub-Riemannian setting:

Corollary 2. Let $M$ be a complete simply connected 3D contact sub-Riemannian manifold. Assume that $\chi=0$ and $\kappa$ is costant on $M$. Then $M$ is isometric to a left-invariant subRiemannian structure. More precisely: 


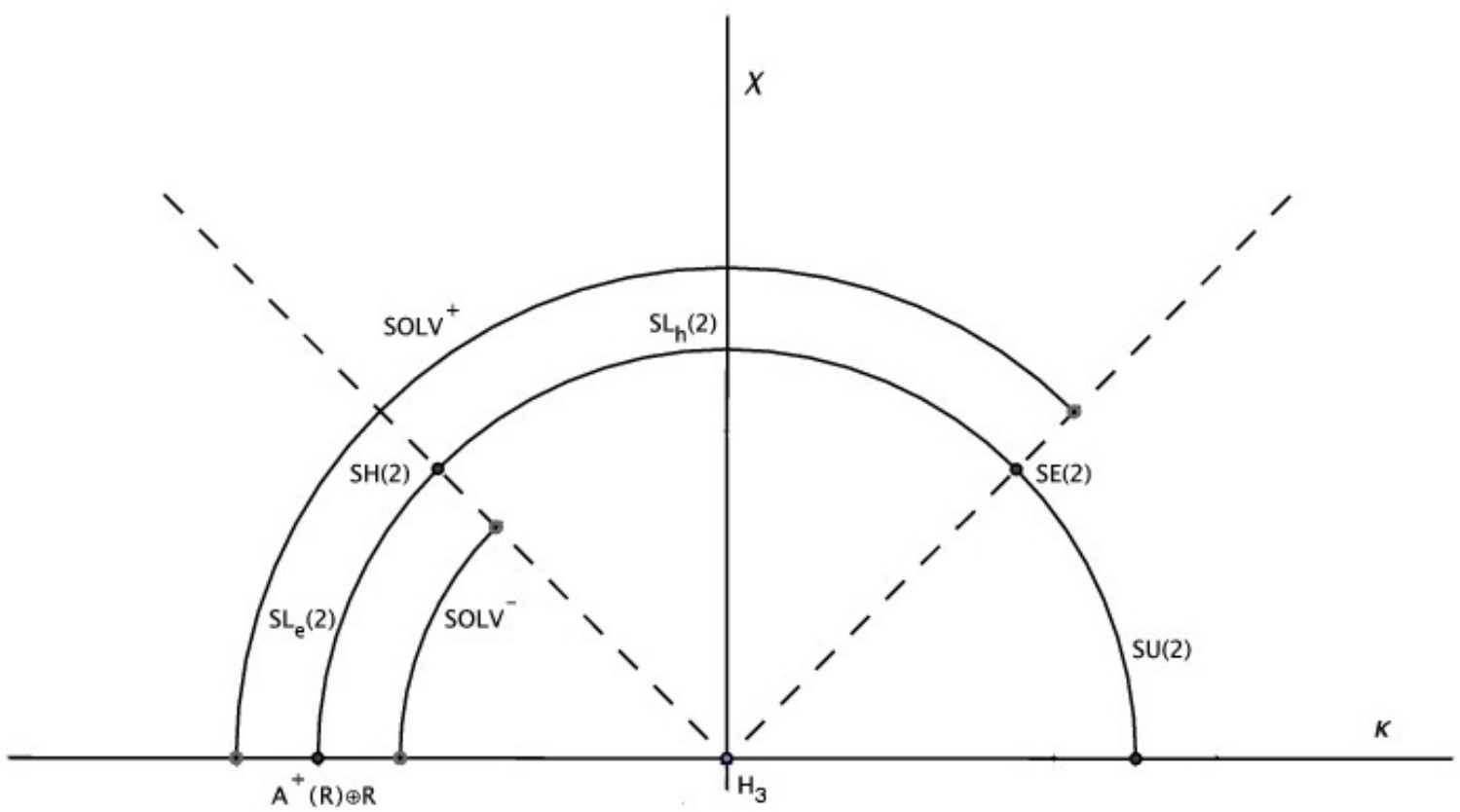

Figure 1: Classification

(i) if $\kappa=0$ it is isometric to the Heisenberg group $H_{3}$,

(ii) if $\kappa=1$ it is isometric to the group $S U(2)$ with Killing metric,

(iii) if $\kappa=-1$ it is isometric to the group $\widetilde{S L}(2)$ with elliptic type Killing metric,

where $\widetilde{S L}(2)$ is the universal covering of $S L(2)$.

Another byproduct of the classification is the fact that there exist non isomorphic Lie groups with locally isometric sub-Riemannian structures. Indeed, as a consequence of Theorem 1, we get that there exists a unique normalized left-invariant structure defined on $A^{+}(\mathbb{R}) \oplus \mathbb{R}$ having $\chi=0, \kappa=-1$. Thus $A^{+}(\mathbb{R}) \oplus \mathbb{R}$ is locally isometric to the group $S L(2)$ with elliptic type Killing metric by Corollary 2 .

This fact was already noted in [13] as a consequence of the classification. In this paper we explicitly compute the global sub-Riemannian isometry between $A^{+}(\mathbb{R}) \oplus \mathbb{R}$ and the universal covering of $S L(2)$ by means of Nagano principle. We then show that this map is well defined on the quotient, giving a global isometry between the group $A^{+}(\mathbb{R}) \times S^{1}$ and the group $S L(2)$, endowed with the sub-Riemannian structure defined by the restriction of the Killing form on the elliptic distribution.

The group $A^{+}(\mathbb{R}) \oplus \mathbb{R}$ can be interpreted as the subgroup of the affine maps on the plane 
that acts as an orientation preserving affinity on one axis and as translations on the other one 1

$$
A^{+}(\mathbb{R}) \oplus \mathbb{R}:=\left\{\left(\begin{array}{lll}
a & 0 & b \\
0 & 1 & c \\
0 & 0 & 1
\end{array}\right), a>0, b, c \in \mathbb{R}\right\} .
$$

The standard left-invariant sub-Riemannian structure on $A^{+}(\mathbb{R}) \oplus \mathbb{R}$ is defined by the orthonormal frame $\Delta=\operatorname{span}\left\{e_{2}, e_{1}+e_{3}\right\}$, where

$$
e_{1}=\left(\begin{array}{lll}
0 & 0 & 1 \\
0 & 0 & 0 \\
0 & 0 & 0
\end{array}\right), \quad e_{2}=\left(\begin{array}{ccc}
-1 & 0 & 0 \\
0 & 0 & 0 \\
0 & 0 & 0
\end{array}\right), \quad e_{3}=\left(\begin{array}{lll}
0 & 0 & 0 \\
0 & 0 & 1 \\
0 & 0 & 0
\end{array}\right),
$$

is a basis of the Lie algebra of the group, satisfying $\left[e_{1}, e_{2}\right]=e_{1}$.

The subgroup $A^{+}(\mathbb{R})$ is topologically homeomorphic to the half-plane $\left\{(a, b) \in \mathbb{R}^{2}, a>0\right\}$ which can be descirbed in standard polar coordinates as $\{(\rho, \theta) \mid \rho>0,-\pi / 2<\theta<\pi / 2\}$.

Theorem 3. The diffeomorphism $\Psi: A^{+}(\mathbb{R}) \times S^{1} \longrightarrow S L(2)$ defined by

$$
\Psi(\rho, \theta, \varphi)=\frac{1}{\sqrt{\rho \cos \theta}}\left(\begin{array}{cc}
\cos \varphi & \sin \varphi \\
\rho \sin (\theta-\varphi) & \rho \cos (\theta-\varphi)
\end{array}\right)
$$

where $(\rho, \theta) \in A^{+}(\mathbb{R})$ and $\varphi \in S^{1}$, is a global sub-Riemannian isometry.

Using this global sub-Riemannian isometry as a change of coordinates one can recover the geometry of the sub-Riemannian structure on the group $A^{+}(\mathbb{R}) \times S^{1}$, starting from the analogous properties of $S L(2)$ (e.g. explicit expression of the sub-Riemannian distance, the cut locus). In particular we notice that, since $A^{+}(\mathbb{R}) \times S^{1}$ is not unimodular, the canonical sub-Laplacian on this group is not expressed as a sum of squares. Indeed if $X_{1}, X_{2}$ denotes the left-invariant vector fields associated to the orthonormal frame, the sub-Laplacian is expressed as follows

$$
L_{s R}=X_{1}^{2}+X_{2}^{2}+X_{1} .
$$

Moreover in the non-unimodular case the generalized Fourier transform method, used in [3], cannot apply . Hence the heat kernel of the corresponding heat equation cannot be computed directly. On the other hand one can use the map (2) to express the solution in terms of the heat kernel on $S L(2)$.

Acknowledgements. The authors are grateful to the anonymous reviewer who noticed some important papers in reference to our work.

\footnotetext{
${ }^{1}$ We can recover the action as an affine map identifying $(x, y) \in \mathbb{R}^{2}$ with $(x, y, 1)^{T}$ and

$$
\left(\begin{array}{lll}
a & 0 & b \\
0 & 1 & c \\
0 & 0 & 1
\end{array}\right)\left(\begin{array}{l}
x \\
y \\
1
\end{array}\right)=\left(\begin{array}{c}
a x+b \\
y+c \\
1
\end{array}\right)
$$
}




\section{Basic definitions}

We start recalling the definition of sub-Riemannian manifold.

Definition 4. A sub-Riemannian manifold is a triple $(M, \Delta, \mathbf{g})$, where

(i) $M$ is a smooth connected $n$-dimensional manifold,

(ii) $\Delta$ is a smooth distribution of constant rank $k<n$, i.e. a smooth map that associates to every $q \in M$ a $k$-dimensional subspace $\Delta_{q}$ of $T_{q} M$,

(iii) $\mathbf{g}_{q}$ is a Riemannian metric on $\Delta_{q}$, that is smooth with respect to $q \in M$.

The set of smooth sections of the distribution

$$
\bar{\Delta}:=\left\{f \in \operatorname{Vec}(M) \mid f(q) \in \Delta_{q}, \forall q \in M\right\} \subset \operatorname{Vec}(M) .
$$

is a subspace of the space of the smooth vector fields on $M$ and its elements are said horizontal vector fields.

A Lipschitz continuous curve $\gamma:[0, T] \rightarrow M$ is admissible (or horizontal) if its derivative is a.e. horizontal, i.e. if $\dot{\gamma}(t) \in \Delta_{\gamma(t)}$ for a.e. $t \in[0, T]$. We denote with $\Omega_{p q}$ the set of admissible paths joining $p$ to $q$.

Given an admissible curve $\gamma$ it is possible to define its lenght

$$
\ell(\gamma)=\int_{0}^{T} \sqrt{\mathbf{g}_{\gamma(t)}(\dot{\gamma}(t), \dot{\gamma}(t))} d t
$$

The Carnot-Caratheodory distance induced by the sub-Riemannian structure is

$$
d(p, q)=\inf \left\{\ell(\gamma), \gamma \in \Omega_{p q}\right\} .
$$

In the following we always assume that the distribution $\Delta$ satisfies the bracket generating condition (also known as Hörmander condition), i.e. the Lie algebra generated by the horizontal vector fields spans at every point the tangent space to the manifold

$$
\operatorname{span}\left\{\left[f_{1}, \ldots,\left[f_{j-1}, f_{j}\right]\right](q), f_{i} \in \bar{\Delta}, j \in \mathbb{N}\right\}=T_{q} M, \quad \forall q \in M .
$$

Under this hypothesis the classical Chow-Rashevsky Theorem [12, 20] implies that $d$ is a well defined metric on $M$ and it induces on $M$ the original topology.

Definition 5. A sub-Riemannian isometry between two sub-Riemannian manifolds $(M, \Delta, \mathbf{g})$ and $\left(N, \Delta^{\prime}, \mathbf{g}^{\prime}\right)$ is a diffeomorphism $\phi: M \rightarrow N$ that satisfies

(i) $\phi_{*}(\Delta)=\Delta^{\prime}$,

(ii) $\mathbf{g}\left(f_{1}, f_{2}\right)=\mathbf{g}^{\prime}\left(\phi_{*} f_{1}, \phi_{*} f_{2}\right), \quad \forall f_{1}, f_{2} \in \bar{\Delta}$.

Definition 6. Let $M$ be a $2 m+1$ dimensional manifold. A sub-Riemannian structure on $M$ is said to be contact if $\Delta$ is a contact distribution, i.e. $\Delta=\operatorname{ker} \omega$, where $\omega \in \Lambda^{1} M$ satisfies $\left(\wedge^{m} d \omega\right) \wedge \omega \neq 0$. Notice that a contact structure is forced to be bracket generating. 
The contact structure endows $M$ with a canonical orientation. On the other hand we will not fix an orientation on the distribution $\Delta$.

Now we briefly recall some facts about sub-Riemannian geodesics. In particular we define the sub-Riemannian Hamiltonian.

Let $M$ be a sub-Riemannian manifold and fix $q_{0} \in M$. We define the endpoint map (at time 1) as

$$
F: \mathcal{U} \rightarrow M, \quad F(\gamma)=\gamma(1),
$$

where $\mathcal{U}$ denotes the set of admissible trajectories starting from $q_{0}$ and defined at time $t=1$. If we fix a point $q_{1} \in M$, the problem of finding shortest paths from $q_{0}$ to $q_{1}$ is equivalent to the following one

$$
\min _{F^{-1}\left(q_{1}\right)} J(\gamma), \quad J(\gamma):=\frac{1}{2} \int_{0}^{1}|\dot{\gamma}(t)|^{2} d t
$$

where $J$ is the action functional. Indeed, it is a standard fact that Cauchy-Schwartz inequality implies that an admissible curve realizes this minimum if and only if it is an arc-lenght parametrized $\ell$-minimizer.

Then the Lagrange multipliers rule implies that any solution of (3) is either a critical point of $F$ or a solution of the equation

$$
\lambda_{1} D_{\gamma} F=d_{\gamma} J, \quad \gamma \in \mathcal{U},
$$

for some $\lambda_{1} \in T_{\gamma(1)}^{*} M$. Solutions of equation (4) are said normal geodesics while critical ponits of $F$ are said abnormal geodesics.

Now we can define the sub-Riemannian Hamiltonian $h \in C^{\infty}\left(T^{*} M\right)$ as follows:

$$
h(\lambda)=\max _{u \in \Delta_{q}}\left\{\langle\lambda, u\rangle-\frac{1}{2}|u|^{2}\right\}, \quad \lambda \in T^{*} M, \quad q=\pi(\lambda),
$$

where $\langle\cdot, \cdot\rangle$ denotes the standard pairing between vectors and covectors. The Pontryagin Maximum Principle gives a perfect characterization of our geodesics. In fact it can be shown that in the contact case there are no abnormal geodesics and a pair $\left(\gamma, \lambda_{1}\right)$ satisfies (4) if and only if there exists a curve $\lambda(t) \in T_{\gamma(t)}^{*} M$ that is a solution of the Hamiltonian system $\dot{\lambda}(t)=\vec{h}(\lambda(t))$ with boundary condition $\lambda(1)=\lambda_{1}$.

Remark 7. Locally the sub-Riemannian structure can be given assigning a set of $k$ smooth linearly independent vector fields that are orthonormal

$$
\Delta_{q}=\operatorname{span}\left\{f_{1}(q), \ldots, f_{k}(q)\right\}, \quad \mathbf{g}_{q}\left(f_{i}(q), f_{j}(q)\right)=\delta_{i j} .
$$

Notice that if we consider a new orthonormal frame which is a rotation of the previous one, we define the same sub-Riemannian structure.

Following this notation a local isometry between two structures defined by the orthonormal frames $\Delta_{M}=\operatorname{span}\left(f_{1}, \ldots, f_{k}\right), \Delta_{N}=\operatorname{span}\left(g_{1}, \ldots, g_{k}\right)$ is given by a local diffeomorphism such that

$$
\phi: M \rightarrow N, \quad \phi_{*}\left(f_{i}\right)=g_{i}, \quad \forall i=1, \ldots, k .
$$


In this setting admissible trajectories are solutions of the equation

$$
\dot{\gamma}(t)=\sum_{i=1}^{k} u_{i}(t) f_{i}(\gamma(t)), \quad \text { for a.e. } t \in[0, T],
$$

for some measurable and bounded control functions $u(t)=\left(u_{1}(t), \ldots, u_{k}(t)\right), u_{i} \in L^{\infty}([0, T])$. Lenght and action of this curve are expressed as follows

$$
\ell(\gamma)=\int_{0}^{T}|u(t)| d t, \quad J(\gamma)=\frac{1}{2} \int_{0}^{T}|u(t)|^{2} d t
$$

where $|\cdot|$ denotes standard Euclidean norm in $\mathbb{R}^{k}$.

Moreover the sub-Riemannian Hamiltonian (5) is written as

$$
h(\lambda)=\frac{1}{2} \sum_{i=1}^{k} h_{i}^{2}(\lambda), \quad \text { where } \quad h_{i}(\lambda)=\left\langle\lambda, f_{i}(q)\right\rangle, \quad q=\pi(\lambda) .
$$

Notice that $h_{i}: T^{*} M \rightarrow \mathbb{R}$ are smooth functions on $T^{*} M$ that are linear on fibers, associated to the vector fields of the frame. The sub-Riemannian Hamiltonian $h$ is a smooth function on $T^{*} M$ which contains all the informations about the sub-Riemannian structure. Indeed it does not depend on the orthonormal frame selected $\left\{f_{1}, \ldots, f_{k}\right\}$, i.e. is invariant for rotations of the frame, and the annichilator of the distribution at a point $\Delta_{q}^{\perp}$ can be recovered as the kernel of the restriction of $h$ to the fiber $T_{q}^{*} M$

$$
\left.\operatorname{ker} h\right|_{T_{q}^{*} M}=\left\{\lambda \in T_{q}^{*} M \mid h_{i}(\lambda)=0, i=1, \ldots, k\right\}=\Delta_{q}^{\perp} .
$$

Remark 8. A sub-Riemannian structure on a Lie group $G$ is said to be left-invariant if

$$
\Delta_{g h}=L_{g *} \Delta_{h}, \quad\langle v, w\rangle_{h}=\left\langle L_{g *} v, L_{g *} w\right\rangle_{g h}, \quad \forall g, h \in G .
$$

where $L_{g}$ denotes the left multiplication map on the group. In particular, to define a leftinvariant structure, it is sufficient to fix a subspace of the Lie algebra $\mathfrak{g}$ of the group and an inner product on it.

We also remark that in this case it is possible to have in (6) a global equality, i.e. to select $k$ globally linearly independent orthonormal vector fields.

\section{Sub-Riemannian invariants}

In this section we study a contact sub-Riemannian structure on a 3D manifold and we give a brief description of its two invariants (see also [5]). We start with the following characterization of contact distributions.

Lemma 9. Let $M$ be a 3D manifold, $\omega \in \Lambda^{1} M$ and $\Delta=\operatorname{ker} \omega$. The following are equivalent:

(i) $\Delta$ is a contact distribution, 
(ii) $\left.d \omega\right|_{\Delta} \neq 0$,

(iii) $\forall f_{1}, f_{2} \in \bar{\Delta}$ linearly independent, then $\left[f_{1}, f_{2}\right] \notin \bar{\Delta}$.

Moreover, in this case, the contact form can be selected in such a way that $\left.d \omega\right|_{\Delta}$ coincide with the Euclidean volume form on $\Delta$.

By Lemma 9 it is not restrictive to assume that the sub-Riemannian structure satisfies:

$$
\begin{gathered}
(M, \omega) \text { is a } 3 \mathrm{D} \text { contact structure, } \\
\Delta=\operatorname{span}\left\{f_{1}, f_{2}\right\}=\operatorname{ker} \omega, \\
\mathbf{g}\left(f_{i}, f_{j}\right)=\delta_{i j}, \quad d \omega\left(f_{1}, f_{2}\right)=1 .
\end{gathered}
$$

We stress that in (7) the orthonormal frame $f_{1}, f_{2}$ is not unique. Indeed every rotated frame (where the angle of rotation depends smoothly on the point) defines the same structure.

The sub-Riemannian Hamiltonian (5) is written

$$
h=\frac{1}{2}\left(h_{1}^{2}+h_{2}^{2}\right) .
$$

Definition 10. In the setting (7) we define the Reeb vector field associated to the contact structure as the unique vector field $f_{0}$ such that

$$
\begin{aligned}
\omega\left(f_{0}\right) & =1, \\
d \omega\left(f_{0}, \cdot\right) & =0 .
\end{aligned}
$$

From the definition it is clear that $f_{0}$ depends only on the sub-Riemannian structure (and its orientation) and not on the frame selected.

Condition (8) is equivalent to

$$
\begin{aligned}
& {\left[f_{1}, f_{0}\right],\left[f_{2}, f_{0}\right] \in \bar{\Delta}} \\
& {\left[f_{2}, f_{1}\right]=f_{0} \quad(\bmod \bar{\Delta}) .}
\end{aligned}
$$

and we deduce the following expression for the Lie algebra of vector fields generated by $f_{0}, f_{1}, f_{2}$

$$
\begin{aligned}
& {\left[f_{1}, f_{0}\right]=c_{01}^{1} f_{1}+c_{01}^{2} f_{2},} \\
& {\left[f_{2}, f_{0}\right]=c_{02}^{1} f_{1}+c_{02}^{2} f_{2},} \\
& {\left[f_{2}, f_{1}\right]=c_{12}^{1} f_{1}+c_{12}^{2} f_{2}+f_{0},}
\end{aligned}
$$

where $c_{i j}^{k}$ are functions on the manifold, called structure constants of the Lie algebra.

If we denote with $\left(\nu_{0}, \nu_{1}, \nu_{2}\right)$ the basis of 1 -form dual to $\left(f_{0}, f_{1}, f_{2}\right)$, we can rewrite $(9)$ as:

$$
\begin{aligned}
& d \nu_{0}=\nu_{1} \wedge \nu_{2}, \\
& d \nu_{1}=c_{01}^{1} \nu_{0} \wedge \nu_{1}+c_{02}^{1} \nu_{0} \wedge \nu_{2}+c_{12}^{1} \nu_{1} \wedge \nu_{2}, \\
& d \nu_{2}=c_{01}^{2} \nu_{0} \wedge \nu_{1}+c_{02}^{2} \nu_{0} \wedge \nu_{2}+c_{12}^{2} \nu_{1} \wedge \nu_{2},
\end{aligned}
$$

Let $h_{0}(\lambda)=\left\langle\lambda, f_{0}(q)\right\rangle$ denote the Hamiltonian linear on fibers associated with the Reeb field $f_{0}$. We now compute the Poisson bracket $\left\{h, h_{0}\right\}$, denoting with $\left\{h, h_{0}\right\}_{q}$ its restriction to the fiber $T_{q}^{*} M$. 
Proposition 11. The Poisson bracket $\left\{h, h_{0}\right\}_{q}$ is a quadratic form. Moreover we have

$$
\begin{gathered}
\left\{h, h_{0}\right\}=c_{01}^{1} h_{1}^{2}+\left(c_{01}^{2}+c_{02}^{1}\right) h_{1} h_{2}+c_{02}^{2} h_{2}^{2}, \\
c_{01}^{1}+c_{02}^{2}=0 .
\end{gathered}
$$

In particular, $\Delta_{q}^{\perp} \subset \operatorname{ker}\left\{h, h_{0}\right\}_{q}$ and $\left\{h, h_{0}\right\}_{q}$ is actually a quadratic form on $T_{q}^{*} M / \Delta_{q}^{\perp}=\Delta_{q}^{*}$.

Proof. Using the equality $\left\{h_{i}, h_{j}\right\}(\lambda)=\left\langle\lambda,\left[f_{i}, f_{j}\right](q)\right\rangle$ we get

$$
\begin{aligned}
\left\{h, h_{0}\right\} & =\frac{1}{2}\left\{h_{1}^{2}+h_{2}^{2}, h_{0}\right\}=h_{1}\left\{h_{1}, h_{0}\right\}+h_{2}\left\{h_{2}, h_{0}\right\} \\
& =h_{1}\left(c_{01}^{1} h_{1}+c_{01}^{2} h_{2}\right)+h_{2}\left(c_{02}^{1} h_{1}+c_{02}^{2} h_{2}\right) \\
& =c_{01}^{1} h_{1}^{2}+\left(c_{01}^{2}+c_{02}^{1}\right) h_{1} h_{2}+c_{02}^{2} h_{2}^{2} .
\end{aligned}
$$

Differentiating the first equation in $(10)$ we find:

$$
\begin{aligned}
0=d^{2} \nu_{0} & =d \nu_{1} \wedge \nu_{2}-\nu_{1} \wedge d \nu_{2} \\
& =\left(c_{01}^{1}+c_{02}^{2}\right) \nu_{0} \wedge \nu_{1} \wedge \nu_{2} .
\end{aligned}
$$

which proves $(12)$.

Being $\left\{h, h_{0}\right\}_{q}$ a quadratic form on the Euclidean plane $\Delta_{q}$ (using the canonical identification of the vector space $\Delta_{q}$ with its dual $\Delta_{q}^{*}$ given by the scalar product), it is a standard fact that it can be interpreted as a symmetric operator on the plane itself. In particular its determinant and its trace are well defined. From $(12)$ we get

$$
\text { trace }\left\{h, h_{0}\right\}_{q}=0 \text {. }
$$

It is natural then to define our first invariant as the positive eigenvalue of this operator, namely:

$$
\chi(q)=\sqrt{-\operatorname{det}\left\{h, h_{0}\right\}_{q}} .
$$

Remark 12 . Notice that, by definition $\chi \geq 0$, and it vanishes everywhere if and only if the flow of the Reeb vector field $f_{0}$ is a flow of sub-Riemannian isometries for $M$.

The second invariant, which was found in [5] as a term of the asymptotic expansion of conjugate locus, is defined in the following way

$$
\kappa(q)=f_{2}\left(c_{12}^{1}\right)-f_{1}\left(c_{12}^{2}\right)-\left(c_{12}^{1}\right)^{2}-\left(c_{12}^{2}\right)^{2}+\frac{c_{01}^{2}-c_{02}^{1}}{2} .
$$

where we refer to notation (9). A direct calculation shows that $\kappa$ is preserved by rotations of the frame $f_{1}, f_{2}$ of the distribution, hence it depends only on the sub-Riemannian structure.

$\chi$ and $\kappa$ are functions defined on the manifold; they reflect intrinsic geometric properties of the sub-Riemannian structure and are preserved by the sub-Riemannian isometries. In particular, $\chi$ and $\kappa$ are constant functions for left-invariant structures on Lie groups (since left translations are isometries). 


\section{Canonical Frames}

In this section we want to show that it is always possible to select a canonical orthonormal frame for the sub-Riemannian structure. In this way we are able to find missing discrete invariants and to classify sub-Riemannian structures simply knowing structure constants $c_{i j}^{k}$ for the canonical frame. We study separately the two cases $\chi \neq 0$ and $\chi=0$.

We start by rewriting and improving Proposition 11 when $\chi \neq 0$.

Proposition 13. Let $M$ be a $3 D$ contact sub-Riemannian manifold and $q \in M$. If $\chi(q) \neq 0$, then there exists a local frame such that

$$
\left\{h, h_{0}\right\}=2 \chi h_{1} h_{2} .
$$

In particular, in the Lie group case with left-invariant stucture, there exists a unique (up to a sign) canonical frame $\left(f_{0}, f_{1}, f_{2}\right)$ such that

$$
\begin{aligned}
& {\left[f_{1}, f_{0}\right]=c_{01}^{2} f_{2},} \\
& {\left[f_{2}, f_{0}\right]=c_{02}^{1} f_{1},} \\
& {\left[f_{2}, f_{1}\right]=c_{12}^{1} f_{1}+c_{12}^{2} f_{2}+f_{0} .}
\end{aligned}
$$

Moreover we have

$$
\chi=\frac{c_{01}^{2}+c_{02}^{1}}{2}, \quad \kappa=-\left(c_{12}^{1}\right)^{2}-\left(c_{12}^{2}\right)^{2}+\frac{c_{01}^{2}-c_{02}^{1}}{2} .
$$

Proof. From Proposition 11 we know that the Poisson bracket $\left\{h, h_{0}\right\}_{q}$ is a non degenerate symmetric operator with zero trace. Hence we have a well defined, up to a sign, orthonormal frame by setting $f_{1}, f_{2}$ as the orthonormal isotropic vectors of this operator (remember that $f_{0}$ depends only on the structure and not on the orthonormal frame on the distribution). It is easily seen that in both of these cases we obtain the expression (15).

Remark 14. Notice that, if we change sign to $f_{1}$ or $f_{2}$, then $c_{12}^{2}$ or $c_{12}^{1}$, respectively, change sign in (16), while $c_{02}^{1}$ and $c_{01}^{2}$ are unaffected. Hence equalities (17) do not depend on the orientation of the sub-Riemannian structure.

If $\chi=0$ the above procedure cannot apply. Indeed both trace and determinant of the operator vanish, hence we have $\left\{h, h_{0}\right\}_{q}=0$. From (11) we get the identities

$$
c_{01}^{1}=c_{02}^{2}=0, \quad c_{01}^{2}+c_{02}^{1}=0 .
$$

so that commutators (9) simplify in (where $c=c_{01}^{2}$ )

$$
\begin{aligned}
& {\left[f_{1}, f_{0}\right]=c f_{2},} \\
& {\left[f_{2}, f_{0}\right]=-c f_{1},} \\
& {\left[f_{2}, f_{1}\right]=c_{12}^{1} f_{1}+c_{12}^{2} f_{2}+f_{0} .}
\end{aligned}
$$

We want to show, with an explicit construction, that also in this case there always exists a rotation of our frame, by an angle that smoothly depends on the point, such that in the new frame $\kappa$ is the only structure constant which appear in $(19)$. 
Lemma 15. Let $f_{1}, f_{2}$ be an orthonormal frame on $M$. If we denote with $\widehat{f_{1}}, \widehat{f_{2}}$ the frame obtained from the previous one with a rotation by an angle $\theta(q)$ and with $\widehat{c}_{i j}^{k}$ structure constants of rotated frame, we have:

$$
\begin{aligned}
& \widehat{c}_{12}^{1}=\cos \theta\left(c_{12}^{1}-f_{1}(\theta)\right)-\sin \theta\left(c_{12}^{2}-f_{2}(\theta)\right), \\
& \widehat{c}_{12}^{2}=\sin \theta\left(c_{12}^{1}-f_{1}(\theta)\right)+\cos \theta\left(c_{12}^{2}-f_{2}(\theta)\right) .
\end{aligned}
$$

Now we can prove the main result of this section.

Proposition 16. Let $M$ be a $3 D$ simply connected contact sub-Riemannian manifold such that $\chi=0$. Then there exists a rotation of the original frame $\widehat{f}_{1}, \widehat{f}_{2}$ such that:

$$
\begin{aligned}
& {\left[\widehat{f}_{1}, f_{0}\right]=\kappa \widehat{f}_{2},} \\
& {\left[\widehat{f}_{2}, f_{0}\right]=-\kappa \widehat{f_{1}},} \\
& {\left[\widehat{f}_{2}, \widehat{f}_{1}\right]=f_{0} .}
\end{aligned}
$$

Proof. Using Lemma 15 we can rewrite the statement in the following way: there exists a function $\theta: M \rightarrow \mathbb{R}$ such that

$$
f_{1}(\theta)=c_{12}^{1}, \quad f_{2}(\theta)=c_{12}^{2} .
$$

Indeed, this would imply $\widehat{c}_{12}^{1}=\widehat{c}_{12}^{2}=0$ and $\kappa=c$.

Let us introduce simplified notations $c_{12}^{1}=\alpha_{1}, c_{12}^{2}=\alpha_{2}$. Then

$$
\kappa=f_{2}\left(\alpha_{1}\right)-f_{1}\left(\alpha_{2}\right)-\left(\alpha_{1}\right)^{2}-\left(\alpha_{2}\right)^{2}+c .
$$

If $\left(\nu_{0}, \nu_{1}, \nu_{2}\right)$ denotes the dual basis to $\left(f_{0}, f_{1}, f_{2}\right)$ we have

$$
d \theta=f_{0}(\theta) \nu_{0}+f_{1}(\theta) \nu_{1}+f_{2}(\theta) \nu_{2} .
$$

and from 190 we get:

$$
\begin{aligned}
f_{0}(\theta) & =\left(\left[f_{2}, f_{1}\right]-\alpha_{1} f_{1}-\alpha_{2} f_{2}\right)(\theta) \\
& =f_{2}\left(\alpha_{1}\right)-f_{1}\left(\alpha_{2}\right)-\alpha_{1}^{2}-\alpha_{2}^{2} \\
& =\kappa-c .
\end{aligned}
$$

Suppose now that (21) are satisfied, we get

$$
d \theta=(\kappa-c) \nu_{0}+\alpha_{1} \nu_{1}+\alpha_{2} \nu_{2}=: \eta
$$

with the r.h.s. independent from $\theta$.

To prove the theorem we have to show that $\eta$ is an exact 1 -form. Since the manifold is simply connected, it is sufficient to prove that $\eta$ is closed. If we denote $\nu_{i j}:=\nu_{i} \wedge \nu_{j}$ dual equations of (19) are:

$$
\begin{aligned}
& d \nu_{0}=\nu_{12}, \\
& d \nu_{1}=-c \nu_{02}+\alpha_{1} \nu_{12}, \\
& d \nu_{2}=c \nu_{01}-\alpha_{2} \nu_{12} .
\end{aligned}
$$


and differentiating we get two nontrivial relations:

$$
\begin{aligned}
& f_{1}(c)+c \alpha_{2}+f_{0}\left(\alpha_{1}\right)=0, \\
& f_{2}(c)-c \alpha_{1}+f_{0}\left(\alpha_{2}\right)=0 .
\end{aligned}
$$

Recollecting all these computations we prove the closure of $\eta$

$$
\begin{aligned}
& d \eta= d(\kappa-c) \wedge \nu_{0}+(\kappa-c) d \nu_{0}+d \alpha_{1} \wedge \nu_{1}+\alpha_{1} d \nu_{1}+d \alpha_{2} \wedge \nu_{2}+\alpha_{2} d \nu_{2} \\
&=-d c \wedge \nu_{0}+(\kappa-c) \nu_{12}+ \\
& \quad+f_{0}\left(\alpha_{1}\right) \nu_{01}-f_{2}\left(\alpha_{1}\right) \nu_{12}+\alpha_{1}\left(\alpha_{1} \nu_{12}-c \nu_{02}\right) \\
& \quad \quad+f_{0}\left(\alpha_{2}\right) \nu_{02}+f_{1}\left(\alpha_{2}\right) \nu_{12}+\alpha_{2}\left(c \nu_{01}-\alpha_{2} \nu_{12}\right) \\
&=\left(f_{0}\left(\alpha_{1}\right)+\alpha_{2} c+f_{1}(c)\right) \nu_{01} \\
& \quad+\left(f_{0}\left(\alpha_{2}\right)-\alpha_{1} c+f_{2}(c)\right) \nu_{02} \\
& \quad \quad+\left(\kappa-c-f_{2}\left(\alpha_{1}\right)+f_{1}\left(\alpha_{2}\right)+\alpha_{1}^{2}+\alpha_{2}^{2}\right) \nu_{12} \\
&=0 .
\end{aligned}
$$

where in the last equality we use 22 and $24-(25)$.

\section{Proof of Theorem 1}

Now we use the results of the previous sections to prove Theorem 1 .

In this section $G$ denotes a $3 \mathrm{D}$ Lie group, with Lie algebra $\mathfrak{g}$, endowed with a left-invariant sub-Riemannian structure defined by the orthonormal frame $f_{1}, f_{2}$, i.e.

$$
\Delta=\operatorname{span}\left\{f_{1}, f_{2}\right\} \subset \mathfrak{g}, \quad \operatorname{span}\left\{f_{1}, f_{2},\left[f_{1}, f_{2}\right]\right\}=\mathfrak{g} .
$$

Recall that for a 3D left-invariant structure to be bracket generating is equivalent to be contact, moreover the Reeb field $f_{0}$ is also a left-invariant vector field by construction.

From the fact that, for left-invariant structures, local invariants are constant functions (see Remark 80 we obtain a necessary condition for two structures to be locally isometric.

Proposition 17. Let $G, H$ be $3 D$ Lie groups with locally isometric sub-Riemannian structures. Then $\chi_{G}=\chi_{H}$ and $\kappa_{G}=\kappa_{H}$.

Notice that this condition is not sufficient. It turns out that there can be up to three mutually non locally isometric normalized structures with the same invariants $\chi, \kappa$.

Remark 18. It is easy to see that $\chi$ and $\kappa$ are homogeneous of degree 2 with respect to dilations of the frame. Indeed assume that the sub-Riemannian structure $(M, \Delta, \mathbf{g})$ is locally defined by the orthonormal frame $f_{1}, f_{2}$, i.e.

$$
\Delta=\operatorname{span}\left\{f_{1}, f_{2}\right\}, \quad \mathbf{g}\left(f_{i}, f_{j}\right)=\delta_{i j} .
$$

Consider now the dilated structure $(M, \Delta, \widetilde{\mathbf{g}})$ defined by the orthonormal frame $\lambda f_{1}, \lambda f_{2}$

$$
\Delta=\operatorname{span}\left\{f_{1}, f_{2}\right\}, \quad \widetilde{\mathbf{g}}\left(f_{i}, f_{j}\right)=\frac{1}{\lambda^{2}} \delta_{i j}, \quad \lambda>0 .
$$


If $\chi, \kappa$ and $\widetilde{\chi}, \widetilde{\kappa}$ denote the invariants of the two structures respectively, we find

$$
\tilde{\chi}=\lambda^{2} \chi, \quad \widetilde{\kappa}=\lambda^{2} \kappa, \quad \lambda>0 .
$$

A dilation of the orthonormal frame corresponds to a multiplication by a factor $\lambda>0$ of all distances in our manifold. Since we are interested in a classification by local isometries, we can always suppose (for a suitable dilation of the orthonormal frame) that the local invariants of our structure satisfy

$$
\chi=\kappa=0, \quad \text { or } \quad \chi^{2}+\kappa^{2}=1,
$$

and we study equivalence classes with respect to local isometries.

Since $\chi$ is non negative by definition (see Remark 12 ), we study separately the two cases $\chi>0$ and $\chi=0$.

\subsection{Case $\chi>0$}

Let $G$ be a 3D Lie group with a left-invariant sub-Riemannian structure such that $\chi \neq 0$. From Proposition 13 we can assume that $\Delta=\operatorname{span}\left\{f_{1}, f_{2}\right\}$ where $f_{1}, f_{2}$ is the canonical frame of the structure. From (16) we obtain the dual equations

$$
\begin{aligned}
& d \nu_{0}=\nu_{1} \wedge \nu_{2}, \\
& d \nu_{1}=c_{02}^{1} \nu_{0} \wedge \nu_{2}+c_{12}^{1} \nu_{1} \wedge \nu_{2}, \\
& d \nu_{2}=c_{01}^{2} \nu_{0} \wedge \nu_{1}+c_{12}^{1} \nu_{1} \wedge \nu_{2} .
\end{aligned}
$$

Using $d^{2}=0$ we obtain structure equations

$$
\left\{\begin{array}{c}
c_{02}^{1} c_{12}^{2}=0, \\
c_{01}^{2} c_{12}^{1}=0 .
\end{array}\right.
$$

We know that the structure constants of the canonical frame are invariant by local isometries (up to change signs of $c_{12}^{1}, c_{12}^{2}$, see Remark 14). Hence, every different choice of coefficients in (16) which satisfy also (27) will belong to a different class of non-isometric structures.

Taking into account that $\chi>0$ implies that $c_{01}^{2}$ and $c_{02}^{1}$ cannot be both non positive (see (17)), we have the following cases:

(i) $c_{12}^{1}=0$ and $c_{12}^{2}=0$. In this first case we get

$$
\begin{aligned}
& {\left[f_{1}, f_{0}\right]=c_{01}^{2} f_{2},} \\
& {\left[f_{2}, f_{0}\right]=c_{02}^{1} f_{1},} \\
& {\left[f_{2}, f_{1}\right]=f_{0},}
\end{aligned}
$$

and formulas (17) imply

$$
\chi=\frac{c_{01}^{2}+c_{02}^{1}}{2}>0, \quad \kappa=\frac{c_{01}^{2}-c_{02}^{1}}{2} .
$$


In addition, we find the relations between the invariants

$$
\chi+\kappa=c_{01}^{2}, \quad \chi-\kappa=c_{02}^{1} .
$$

We have the following subcases:

(a) If $c_{02}^{1}=0$ we get the Lie algebra $\mathfrak{s e}(2)$ of the group $S E(2)$ of the Euclidean isometries of $\mathbb{R}^{2}$, and it holds $\chi=\kappa$.

(b) If $c_{01}^{2}=0$ we get the Lie algebra $\mathfrak{s h}(2)$ of the group $S H(2)$ of the Hyperbolic isometries of $\mathbb{R}^{2}$, and it holds $\chi=-\kappa$.

(c) If $c_{01}^{2}>0$ and $c_{02}^{1}<0$ we get the Lie algebra $\mathfrak{s u}(2)$ and $\chi-\kappa<0$.

(d) If $c_{01}^{2}<0$ and $c_{02}^{1}>0$ we get the Lie algebra $\mathfrak{s l}(2)$ with $\chi+\kappa<0$.

(e) If $c_{01}^{2}>0$ and $c_{02}^{1}>0$ we get the Lie algebra $\mathfrak{s l}(2)$ with $\chi+\kappa>0, \chi-\kappa>0$.

(ii) $c_{02}^{1}=0$ and $c_{12}^{1}=0$. In this case we have

$$
\begin{aligned}
& {\left[f_{1}, f_{0}\right]=c_{01}^{2} f_{2},} \\
& {\left[f_{2}, f_{0}\right]=0,} \\
& {\left[f_{2}, f_{1}\right]=c_{12}^{2} f_{2}+f_{0},}
\end{aligned}
$$

and necessarily $c_{01}^{2} \neq 0$. Moreover we get

$$
\chi=\frac{c_{01}^{2}}{2}>0, \quad \kappa=-\left(c_{12}^{2}\right)^{2}+\frac{c_{01}^{2}}{2},
$$

from which it follows

$$
\chi-\kappa \geq 0 .
$$

The Lie algebra $\mathfrak{g}=\operatorname{span}\left\{f_{1}, f_{2}, f_{3}\right\}$ defined by (28) satisfies $\operatorname{dim}[\mathfrak{g}, \mathfrak{g}]=2$, hence it can be interpreted as the operator $A=\operatorname{ad} f_{1}$ which acts on the subspace $\operatorname{span}\left\{f_{0}, f_{2}\right\}$. Moreover, it can be easily computed that

$$
\text { trace } A=-c_{12}^{2}, \quad \operatorname{det} A=c_{01}^{2}>0,
$$

and we can find the useful relation

$$
2 \frac{\operatorname{trace}^{2} A}{\operatorname{det} A}=1-\frac{\kappa}{\chi}
$$

(iii) $c_{01}^{2}=0$ and $c_{12}^{2}=0$. In this last case we get

$$
\begin{aligned}
& {\left[f_{1}, f_{0}\right]=0,} \\
& {\left[f_{2}, f_{0}\right]=c_{02}^{1} f_{1},} \\
& {\left[f_{2}, f_{1}\right]=c_{12}^{1} f_{1}+f_{0},}
\end{aligned}
$$


and $c_{02}^{1} \neq 0$. Moreover we get

$$
\chi=\frac{c_{02}^{1}}{2}>0, \quad \kappa=-\left(c_{12}^{1}\right)^{2}-\frac{c_{02}^{1}}{2},
$$

from which it follows

$$
\chi+\kappa \leq 0 .
$$

As before, the Lie algebra $\mathfrak{g}=\operatorname{span}\left\{f_{1}, f_{2}, f_{3}\right\}$ defined by (30) has two-dimensional square and it can be interpreted as the operator $A=$ ad $f_{2}$ which acts on the plane $\operatorname{span}\left\{f_{0}, f_{1}\right\}$. It can be easily seen that it holds

$$
\text { trace } A=c_{12}^{1}, \quad \operatorname{det} A=-c_{02}^{1}<0,
$$

and we have an analogous relation

$$
2 \frac{\operatorname{trace}^{2} A}{\operatorname{det} A}=1+\frac{\kappa}{\chi}
$$

Remark 19. Lie algebras of cases (ii) and (iii) are solvable algebras and we will denote respectively $\mathfrak{s o l v} \mathfrak{v}^{+}$and $\mathfrak{s o l v} \mathfrak{v}^{-}$, where the sign depends on the determinant of the operator it represents. In particular, formulas (29) and (31) permits to recover the ratio between invariants (hence to determine a unique normalized structure) only from intrinsic properties of the operator. Notice that if $c_{12}^{2}=0$ we recover the normalized structure $(i)-(a)$ while if $c_{12}^{1}=0$ we get the case $(i)-(b)$.

Remark 20. The algebra $\mathfrak{s l}(2)$ is the only case where we can define two nonequivalent distributions which corresponds to the case that Killing form restricted on the distribution is positive definite (case $(d))$ or indefinite (case $(e))$. We will refer to the first one as the elliptic structure on $\mathfrak{s l}(2)$, denoted $\mathfrak{s l}_{e}(2)$, and with hyperbolic structure in the other case, denoting $\mathfrak{s l}_{h}(2)$.

\subsection{Case $\chi=0$}

A direct consequence of Proposition 16 for left-invariant structures is the following

Corollary 21. Let $G, H$ be Lie groups with left-invariant sub-Riemannian structures and assume $\chi_{G}=\chi_{H}=0$. Then $G$ and $H$ are locally isometric if and only if $\kappa_{G}=\kappa_{H}$.

Thanks to this result it is very easy to complete our classification. Indeed it is sufficient to find all left-invariant structures such that $\chi=0$ and to compare their second invariant $\kappa$.

A straightforward calculation leads to the following list of the left-invariant structures on simply connected three dimensional Lie groups with $\chi=0$ :

- $H_{3}$ is the Heisenberg nilpotent group; then $\kappa=0$.

- $S U(2)$ with the Killing inner product; then $\kappa>0$.

- $\widetilde{S L}(2)$ with the elliptic distribution and Killing inner product; then $\kappa<0$. 
- $A^{+}(\mathbb{R}) \oplus \mathbb{R} ;$ then $\kappa<0$.

Remark 22. In particular, we have the following:

(i) All left-invariant sub-Riemannian structures on $H_{3}$ are locally isometric,

(ii) There exists on $A^{+}(\mathbb{R}) \oplus \mathbb{R}$ a unique (modulo dilations) left-invariant sub-Riemannian structure, which is locally isometric to $S L_{e}(2)$ with the Killing metric.

Proof of Theorem 1 is now completed and we can recollect our result as in Figure 1, where we associate to every normalized structure a point in the $(\kappa, \chi)$ plane: either $\chi=\kappa=0$, or $(\kappa, \chi)$ belong to the semicircle

$$
\left\{(\kappa, \chi) \in \mathbb{R}^{2}, \chi^{2}+\kappa^{2}=1, \chi>0\right\} .
$$

Notice that different points means that sub-Riemannian structures are not locally isometric.

\section{Proof of Theorem 3}

In this section we want to write explicitly the sub-Riemannian isometry between $S L(2)$ and $A^{+}(\mathbb{R}) \times S^{1}$.

Consider the Lie algebra $\mathfrak{s l}(2)=\left\{A \in M_{2}(\mathbb{R}), \operatorname{trace}(A)=0\right\}=\operatorname{span}\left\{g_{1}, g_{2}, g_{3}\right\}$, where

$$
g_{1}=\frac{1}{2}\left(\begin{array}{cc}
1 & 0 \\
0 & -1
\end{array}\right), \quad g_{2}=\frac{1}{2}\left(\begin{array}{ll}
0 & 1 \\
1 & 0
\end{array}\right), \quad g_{3}=\frac{1}{2}\left(\begin{array}{cc}
0 & 1 \\
-1 & 0
\end{array}\right)
$$

The sub-Riemannian structure on $S L(2)$ defined by the Killing form on the elliptic distribution is given by the orthonormal frame

$$
\Delta_{\mathfrak{s l}}=\operatorname{span}\left\{g_{1}, g_{2}\right\}, \quad \text { and } \quad g_{0}:=-g_{3},
$$

is the Reeb vector field. Notice that this frame is already canonical since equations 20 are satisfied. Indeed

$$
\left[g_{1}, g_{0}\right]=-g_{2}=\kappa g_{2} .
$$

Recall that the universal covering of $S L(2)$, which we denote $\widetilde{S L}(2)$, is a simply connected Lie group with Lie algebra $\mathfrak{s l}(2)$. Hence $(32)$ define a left-invariant structure also on the universal covering.

On the other hand we consider the following coordinates on the Lie group $A^{+}(\mathbb{R}) \oplus \mathbb{R}$, that are well-adapted for our further calculations

$$
A^{+}(\mathbb{R}) \oplus \mathbb{R}:=\left\{\left(\begin{array}{ccc}
-y & 0 & x \\
0 & 1 & z \\
0 & 0 & 1
\end{array}\right), \quad y<0, x, z \in \mathbb{R}\right\} .
$$

It is easy to see that, in these coordinates, the group law reads

$$
(x, y, z)\left(x^{\prime}, y^{\prime}, z^{\prime}\right)=\left(x-y x^{\prime},-y y^{\prime}, z+z^{\prime}\right)
$$


and its Lie algebra $\mathfrak{a}(\mathbb{R}) \oplus \mathbb{R}$ is generated by the vector fields

$$
e_{1}=-y \partial_{x}, \quad e_{2}=-y \partial_{y}, \quad e_{3}=\partial_{z},
$$

with the only nontrivial commutator relation $\left[e_{1}, e_{2}\right]=e_{1}$.

The left-invariant structure on $A^{+}(\mathbb{R}) \oplus \mathbb{R}$ is defined by the orthonormal frame

$$
\begin{aligned}
\Delta_{\mathfrak{a}} & =\operatorname{span}\left\{f_{1}, f_{2}\right\}, \\
f_{1} & :=e_{2}=-y \partial_{y}, \\
f_{2} & :=e_{1}+e_{3}=-y \partial_{x}+\partial_{z} .
\end{aligned}
$$

With straightforward calculations we compute the Reeb vector field $f_{0}=-e_{3}=-\partial_{z}$.

This frame is not canonical since it does not satisfy equations 20. Hence we can apply Proposition 16 to find the canonical frame, that will be no more left-invariant.

Following the notation of Proposition 16 we have

Lemma 23. The canonical orthonormal frame on $A^{+}(\mathbb{R}) \oplus \mathbb{R}$ has the form:

$$
\begin{aligned}
& \widehat{f_{1}}=y \sin z \partial_{x}-y \cos z \partial_{y}-\sin z \partial_{z}, \\
& \widehat{f_{2}}=-y \cos z \partial_{x}-y \sin z \partial_{y}+\cos z \partial_{z} .
\end{aligned}
$$

Proof. It is equivalent to show that the rotation defined in the proof of Proposition 16 is $\theta(x, y, z)=z$. The dual basis to our frame $\left\{f_{1}, f_{2}, f_{0}\right\}$ is given by

$$
\nu_{1}=-\frac{1}{y} d y, \quad \nu_{2}=-\frac{1}{y} d x, \quad \nu_{0}=-\frac{1}{y} d x-d z .
$$

Moreover we have $\left[f_{1}, f_{0}\right]=\left[f_{2}, f_{0}\right]=0$ and $\left[f_{2}, f_{1}\right]=f_{2}+f_{0}$ so that, in equation (23) we get $c=0, \alpha_{1}=0, \alpha_{2}=1$. Hence

$$
d \theta=-\nu_{0}+\nu_{2}=d z
$$

Now we have two canonical frames $\left\{\widehat{f}_{1}, \widehat{f}_{2}, f_{0}\right\}$ and $\left\{g_{1}, g_{2}, g_{0}\right\}$, whose Lie algebras satisfy the same commutator relations:

$$
\begin{aligned}
{\left[\widehat{f}_{1}, f_{0}\right] } & =-\widehat{f}_{2}, & {\left[g_{1}, g_{0}\right] } & =-g_{2}, \\
{\left[\widehat{f}_{2}, f_{0}\right] } & =\widehat{f}_{1}, & {\left[g_{2}, g_{0}\right] } & =g_{1}, \\
{\left[\widehat{f}_{2}, \widehat{f}_{1}\right] } & =f_{0}, & {\left[g_{2}, g_{1}\right] } & =0 .
\end{aligned}
$$

Let us consider the two control systems

$$
\begin{aligned}
& \dot{q}=u_{1} \widehat{f}_{1}(q)+u_{2} \widehat{f}_{2}(q)+u_{0} f_{0}(q), \quad q \in A^{+}(\mathbb{R}) \oplus \mathbb{R}, \\
& \dot{x}=u_{1} g_{1}(x)+u_{2} g_{2}(x)+u_{0} g_{0}(x), \quad x \in \widetilde{S L}(2) .
\end{aligned}
$$


and denote with $x_{u}(t), q_{u}(t), t \in[0, T]$ the solutions of the equations relative to the same control $u=\left(u_{1}, u_{2}, u_{0}\right)$. Nagano Principle (see [7] and also [19, 24, 25]) ensure that the map

$$
\widetilde{\Psi}: A^{+}(\mathbb{R}) \oplus \mathbb{R} \rightarrow \widetilde{S L}(2), \quad q_{u}(T) \mapsto x_{u}(T) .
$$

that sends the final point of the first system to the final point of the second one, is well-defined and does not depend on the control $u$.

Thus we can find the endpoint map of both systems relative to constant controls, i.e. considering maps

$$
\begin{array}{ll}
\widetilde{F}: \mathbb{R}^{3} \rightarrow A^{+}(\mathbb{R}) \oplus \mathbb{R}, & \left(t_{1}, t_{2}, t_{0}\right) \mapsto e^{t_{0} f_{0}} \circ e^{t_{2} \widehat{f}_{2}} \circ e^{t_{1} \widehat{f}_{1}}\left(1_{A}\right), \\
\widetilde{G}: \mathbb{R}^{3} \rightarrow S L(2), & \left(t_{1}, t_{2}, t_{0}\right) \mapsto e^{t_{0} g_{0}} \circ e^{t_{2} g_{2}} \circ e^{t_{1} g_{1}}\left(1_{S L}\right) .
\end{array}
$$

where we denote with $1_{A}$ and $1_{S L}$ identity element of $A^{+}(\mathbb{R}) \oplus \mathbb{R}$ and $\widetilde{S L}(2)$, respectively.

The composition of these two maps makes the following diagram commutative

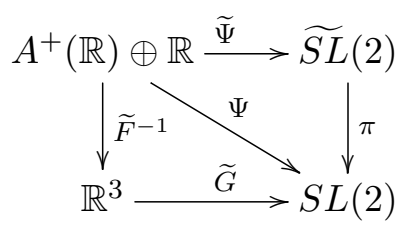

where $\pi: \widetilde{S L}(2) \rightarrow S L(2)$ is the canonical projection and we set $\Psi:=\pi \circ \widetilde{\Psi}$.

To simplify computation we introduce the rescaled maps

$$
F(t):=\widetilde{F}(2 t), \quad G(t):=\widetilde{G}(2 t), \quad t=\left(t_{1}, t_{2}, t_{0}\right),
$$

and solving differential equations we get from (38) the following expressions

$$
F\left(t_{1}, t_{2}, t_{0}\right)=\left(2 e^{-2 t_{1}} \frac{\tanh t_{2}}{1+\tanh ^{2} t_{2}},-e^{-2 t_{1}} \frac{1-\tanh ^{2} t_{2}}{1+\tanh ^{2} t_{2}}, 2\left(\arctan \left(\tanh t_{2}\right)-t_{0}\right)\right) .
$$

The function $F$ is globally invertible on its image and its inverse

$$
F^{-1}(x, y, z)=\left(-\frac{1}{2} \log \sqrt{x^{2}+y^{2}}, \operatorname{arctanh}\left(\frac{y+\sqrt{x^{2}+y^{2}}}{x}\right), \arctan \left(\frac{y+\sqrt{x^{2}+y^{2}}}{x}\right)-\frac{z}{2}\right) .
$$

is defined for every $y<0$ and for every $x$ (it is extended by continuity at $x=0$ ).

On the other hand, the map (39) can be expressed by the product of exponential matrices as follow: 2

$$
G\left(t_{1}, t_{2}, t_{0}\right)=\left(\begin{array}{cc}
e^{t_{1}} & 0 \\
0 & e^{-t_{2}}
\end{array}\right)\left(\begin{array}{cc}
\cosh t_{2} & \sinh t_{2} \\
\sinh t_{2} & \cosh t_{2}
\end{array}\right)\left(\begin{array}{cc}
\cos t_{0} & -\sin t_{0} \\
\sin t_{0} & \cos t_{0}
\end{array}\right)
$$

\footnotetext{
${ }^{2}$ since we consider left-invariant system, we must multiply matrices on the right.
} 
To simplify the computations, we consider standard polar coordinates $(\rho, \theta)$ on the halfplane $\{(x, y), y<0\}$, where $-\pi / 2<\theta<\pi / 2$ is the angle that the point $(x, y)$ defines with $y$-axis. In particular, it is easy to see that the expression that appear in $F^{-1}$ is naturally related to these coordinates:

$$
\xi=\xi(\theta):=\tan \frac{\theta}{2}= \begin{cases}\frac{y+\sqrt{x^{2}+y^{2}}}{x}, & \text { if } x \neq 0, \\ 0, & \text { if } x=0 .\end{cases}
$$

Hence we can rewrite

$$
F^{-1}(\rho, \theta, z)=\left(-\frac{1}{2} \log \rho, \operatorname{arctanh} \xi, \arctan \xi-\frac{z}{2}\right) .
$$

and compute the composition $\Psi=G \circ F^{-1}: A^{+}(\mathbb{R}) \oplus \mathbb{R} \longrightarrow S L(2)$. Once we substitute these expressions in 42 , the third factor is a rotation matrix by an angle $\arctan \xi-z / 2$. Splitting this matrix in two consecutive rotations and using standard trigonometric identities $\cos (\arctan \xi)=\frac{1}{\sqrt{1+\xi^{2}}}, \sin (\arctan \xi)=\frac{\xi}{\sqrt{1+\xi^{2}}}, \cosh (\operatorname{arctanh} \xi)=\frac{1}{\sqrt{1-\xi^{2}}}, \sinh (\operatorname{arctanh} \xi)=$ $\frac{\xi}{\sqrt{1-\xi^{2}}}$, for $\xi \in(-1,1)$, we obtain:

$$
\begin{aligned}
& \Psi(\rho, \theta, z)= \\
& =\left(\begin{array}{cc}
\rho^{-1 / 2} & 0 \\
0 & \rho^{1 / 2}
\end{array}\right)\left(\begin{array}{cc}
\frac{1}{\sqrt{1-\xi^{2}}} & \frac{\xi}{\sqrt{1-\xi^{2}}} \\
\frac{\xi}{\sqrt{1-\xi^{2}}} & \frac{1}{\sqrt{1-\xi^{2}}}
\end{array}\right)\left(\begin{array}{cc}
\frac{1}{\sqrt{1+\xi^{2}}} & -\frac{\xi}{\sqrt{1+\xi^{2}}} \\
\frac{\xi}{\sqrt{1+\xi^{2}}} & \frac{1}{\sqrt{1+\xi^{2}}}
\end{array}\right)\left(\begin{array}{cc}
\cos \frac{z}{2} & \sin \frac{z}{2} \\
-\sin \frac{z}{2} & \cos \frac{z}{2}
\end{array}\right) .
\end{aligned}
$$


Then using identities: $\cos \theta=\frac{1-\xi^{2}}{1+\xi^{2}}, \sin \theta=\frac{2 \xi}{1+\xi^{2}}$, we get

$$
\begin{aligned}
\Psi(\rho, \theta, z) & =\left(\begin{array}{cc}
\rho^{-1 / 2} & 0 \\
0 & \rho^{1 / 2}
\end{array}\right)\left(\begin{array}{cc}
\frac{1+\xi^{2}}{\sqrt{1-\xi^{4}}} & 0 \\
\frac{2 \xi}{\sqrt{1-\xi^{4}}} & \frac{1-\xi^{2}}{\sqrt{1-\xi^{4}}}
\end{array}\right)\left(\begin{array}{cc}
\cos \frac{z}{2} & \sin \frac{z}{2} \\
-\sin \frac{z}{2} & \cos \frac{z}{2}
\end{array}\right) \\
& =\sqrt{\frac{1+\xi^{2}}{1-\xi^{2}}}\left(\begin{array}{cc}
\rho^{-1 / 2} & 0 \\
0 & \rho^{1 / 2}
\end{array}\right)\left(\begin{array}{cc}
1 & 0 \\
\frac{2 \xi}{1+\xi^{2}} & \frac{1-\xi^{2}}{1+\xi^{2}}
\end{array}\right)\left(\begin{array}{cc}
\cos \frac{z}{2} & \sin \frac{z}{2} \\
-\sin \frac{z}{2} & \cos \frac{z}{2}
\end{array}\right) \\
& =\frac{1}{\sqrt{\rho \cos \theta}}\left(\begin{array}{cc}
1 & 0 \\
0 & \rho
\end{array}\right)\left(\begin{array}{cc}
1 & 0 \\
\sin \theta & \cos \theta
\end{array}\right)\left(\begin{array}{cc}
\cos \frac{z}{2} & \sin \frac{z}{2} \\
-\sin \frac{z}{2} & \cos \frac{z}{2}
\end{array}\right) \\
& =\frac{1}{\sqrt{\rho \cos \theta}}\left(\begin{array}{cc}
\cos \frac{z}{2} & \sin \frac{z}{2} \\
\rho \sin \left(\theta-\frac{z}{2}\right) & \rho \cos \left(\theta-\frac{z}{2}\right)
\end{array}\right) .
\end{aligned}
$$

Lemma 24. The set $\Psi^{-1}(I)$ is a normal subgroup of $A^{+}(\mathbb{R}) \oplus \mathbb{R}$.

Proof. It is easy to show that $\Psi^{-1}(I)=\{F(0,0,2 k \pi), k \in \mathbb{Z}\}$. From (41) we see that $F(0,0,2 k \pi)=(0,-1,-4 k \pi)$ and $(33)$ implies that this is a normal subgroup. Indeed it is enoough to prove that $\Psi^{-1}(I)$ is a subgroup of the centre, that follows from the identity

$$
\left(\begin{array}{ccc}
1 & 0 & 0 \\
0 & 1 & 4 k \pi \\
0 & 0 & 1
\end{array}\right)\left(\begin{array}{ccc}
-y & 0 & x \\
0 & 1 & z \\
0 & 0 & 1
\end{array}\right)=\left(\begin{array}{ccc}
-y & 0 & x \\
0 & 1 & z+4 k \pi \\
0 & 0 & 1
\end{array}\right)=\left(\begin{array}{ccc}
-y & 0 & x \\
0 & 1 & z \\
0 & 0 & 1
\end{array}\right)\left(\begin{array}{ccc}
1 & 0 & 0 \\
0 & 1 & 4 k \pi \\
0 & 0 & 1
\end{array}\right) .
$$

Remark 25. With a standard topological argument it is possible to prove that actually $\Psi^{-1}(A)$ is a discrete countable set for every $A \in S L(2)$, and $\Psi$ is a representation of $A^{+}(\mathbb{R}) \oplus \mathbb{R}$ as universal covering of $S L(2)$.

By Lemma 24 the map $\Psi$ is well defined isomorphism between the quotient

$$
\frac{A^{+}(\mathbb{R}) \oplus \mathbb{R}}{\Psi^{-1}(I)} \simeq A^{+}(\mathbb{R}) \times S^{1}
$$

and the group $S L(2)$, defined by restriction of $\Psi$ on $z \in[-2 \pi, 2 \pi]$. 
If we consider the new variable $\varphi=z / 2$, defined on $[-\pi, \pi]$, we can finally write the global isometry as

$$
\Psi(\rho, \theta, \varphi)=\frac{1}{\sqrt{\rho \cos \theta}}\left(\begin{array}{cc}
\cos \varphi & \sin \varphi \\
\rho \sin (\theta-\varphi) & \rho \cos (\theta-\varphi)
\end{array}\right)
$$

where $(\rho, \theta) \in A^{+}(\mathbb{R})$ and $\varphi \in S^{1}$.

Remark 26. In the coordinate set defined above we have that $1_{A}=(1,0,0)$ and

$$
\Psi\left(1_{A}\right)=\Psi(1,0,0)=\left(\begin{array}{ll}
1 & 0 \\
0 & 1
\end{array}\right)=1_{S L} .
$$

On the other hand $\Psi$ is not a homomorphism since in $A^{+}(\mathbb{R}) \oplus \mathbb{R}$ it holds

$$
\left(\frac{\sqrt{2}}{2}, \frac{\pi}{4}, \pi\right)\left(\frac{\sqrt{2}}{2},-\frac{\pi}{4},-\pi\right)=1_{A}
$$

while it can be easily checked from 43 that

$$
\Psi\left(\frac{\sqrt{2}}{2}, \frac{\pi}{4}, \pi\right) \Psi\left(\frac{\sqrt{2}}{2},-\frac{\pi}{4},-\pi\right)=\left(\begin{array}{cc}
2 & 0 \\
1 / 2 & 1 / 2
\end{array}\right) \neq 1_{S L}
$$

\section{References}

[1] A. Agrachev, D. Barilari, and U. Boscain. On the Hausdorff volume in sub-Riemannian geometry. Calculus of Variations and Partial Differential Equations, pages 1-34, 2011. 10.1007/s00526-0110414-y.

[2] A. Agrachev, D. Barilari, and U. Boscain. Introduction to Riemannian and sub-Riemannian geometry (Lecture Notes), http://people.sissa.it/agrachev/agrachev_files/notes.html. 2012.

[3] A. Agrachev, U. Boscain, J.-P. Gauthier, and F. Rossi. The intrinsic hypoelliptic Laplacian and its heat kernel on unimodular Lie groups. J. Funct. Anal., 256(8):2621-2655, 2009.

[4] A. A. Agrachev. Methods of control theory in nonholonomic geometry. In Proceedings of the International Congress of Mathematicians, Vol. 1, 2 (Zürich, 1994), pages 1473-1483. Birkhäuser, Basel, 1995.

[5] A. A. Agrachev. Exponential mappings for contact sub-Riemannian structures. J. Dynam. Control Systems, 2(3):321-358, 1996.

[6] A. A. Agrachev, G. Charlot, J. P. A. Gauthier, and V. M. Zakalyukin. On sub-Riemannian caustics and wave fronts for contact distributions in the three-space. J. Dynam. Control Systems, 6(3):365$395,2000$.

[7] A. A. Agrachev and Y. L. Sachkov. Control theory from the geometric viewpoint, volume 87 of Encyclopaedia of Mathematical Sciences. Springer-Verlag, Berlin, 2004. Control Theory and Optimization, II.

[8] A. Bellaïche. The tangent space in sub-Riemannian geometry. J. Math. Sci. (New York), 83(4):461476, 1997. Dynamical systems, 3. 
[9] U. Boscain and F. Rossi. Invariant Carnot-Caratheodory metrics on $S^{3}, \mathrm{SO}(3), \mathrm{SL}(2)$, and lens spaces. SIAM J. Control Optim., 47(4):1851-1878, 2008.

[10] É. Cartan. Sur la géométrie pseudo-conforme des hypersurfaces de l'espace de deux variables complexes II. Ann. Scuola Norm. Sup. Pisa Cl. Sci. (2), 1(4):333-354, 1932.

[11] É. Cartan. Sur la géométrie pseudo-conforme des hypersurfaces de l'espace de deux variables complexes. Ann. Mat. Pura Appl., 11(1):17-90, 1933.

[12] W.-L. Chow. Über Systeme von linearen partiellen Differentialgleichungen erster Ordnung. Math. Ann., 117:98-105, 1939.

[13] E. Falbel and C. Gorodski. Sub-Riemannian homogeneous spaces in dimensions 3 and 4. Geom. Dedicata, 62(3):227-252, 1996.

[14] V. Gershkovich and A. Vershik. Nonholonomic manifolds and nilpotent analysis. J. Geom. Phys., $5(3): 407-452,1988$.

[15] M. Gromov. Carnot-Carathéodory spaces seen from within. In Sub-Riemannian geometry, volume 144 of Progr. Math., pages 79-323. Birkhäuser, Basel, 1996.

[16] N. Jacobson. Lie algebras. Interscience Tracts in Pure and Applied Mathematics, No. 10. Interscience Publishers (a division of John Wiley \& Sons), New York-London, 1962.

[17] I. Moiseev and Y. L. Sachkov. Maxwell strata in sub-Riemannian problem on the group of motions of a plane. ESAIM Control Optim. Calc. Var., 16:380-399, 2010.

[18] R. Montgomery. A tour of subriemannian geometries, their geodesics and applications, volume 91 of Mathematical Surveys and Monographs. American Mathematical Society, Providence, RI, 2002.

[19] T. Nagano. Linear differential systems with singularities and an application to transitive Lie algebras. J. Math. Soc. Japan, 18:398-404, 1966.

[20] P. Rashevsky. Any two points of a totally nonholonomic space may be connected by an admissible line. Uch. Zap. Ped Inst. im. Liebknechta, 2:83-84, 1938.

[21] Y. L. Sachkov. Conjugate and cut time in the sub-Riemannian problem on the group of motions of a plane. ESAIM Control Optim. Calc. Var., 16:1018-1039, 2010.

[22] R. S. Strichartz. Sub-Riemannian geometry. J. Differential Geom., 24(2):221-263, 1986.

[23] R. S. Strichartz. Corrections to: "Sub-Riemannian geometry". J. Differential Geom., 30(2):595$596,1989$.

[24] H. J. Sussmann. An extension of a theorem of Nagano on transitive Lie algebras. Proc. Amer. Math. Soc., 45:349-356, 1974.

[25] H. J. Sussmann. Lie brackets, real analyticity and geometric control. In Differential geometric control theory (Houghton, Mich., 1982), volume 27 of Progr. Math., pages 1-116. Birkhäuser Boston, Boston, MA, 1983. 